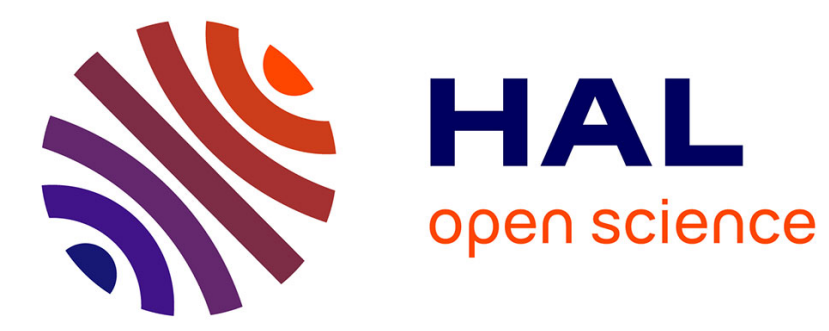

\title{
The motion of a freely falling chain tip
}

W Tomaszewski, P Pieranski, J.-C Géminard

\section{To cite this version:}

W Tomaszewski, P Pieranski, J.-C Géminard. The motion of a freely falling chain tip. American Journal of Physics, 2005, 10.1119/1.2204074 . hal-01238243

\section{HAL Id: hal-01238243 \\ https://hal.science/hal-01238243}

Submitted on 4 Dec 2015

HAL is a multi-disciplinary open access archive for the deposit and dissemination of scientific research documents, whether they are published or not. The documents may come from teaching and research institutions in France or abroad, or from public or private research centers.
L'archive ouverte pluridisciplinaire HAL, est destinée au dépôt et à la diffusion de documents scientifiques de niveau recherche, publiés ou non, émanant des établissements d'enseignement et de recherche français ou étrangers, des laboratoires publics ou privés. 


\title{
The motion of a freely falling chain tip
}

\author{
W. Tomaszewski ${ }^{\mathrm{a})}$ and P. Pieranski ${ }^{\mathrm{b})}$ \\ Poznan University of Technology, Nieszawska 13A, 60-965 Poznan, Poland \\ J.-C. Geminard ${ }^{\text {c) }}$ \\ Ecole Normale Supérieure de Lyon, Laboratoire de Physique, 46 Allée d'Italie, 69364 Lyon Cedex 07, \\ France
}

(Received 4 October 2005; accepted 14 April 2006)

\begin{abstract}
The dynamics of the tip of a falling chain is analyzed. The results of laboratory experiments are presented and compared with the results of numerical simulations. The time dependence of the velocity and the acceleration of the chain tip for several initial conformations of the chain are determined. A simple analytical model of the system is also considered. (C) 2006 American Association of Physics Teachers.
\end{abstract}

[DOI: $10.1119 / 1.2204074]$

\section{INTRODUCTION}

The problem of bodies falling in a gravitational field is so old that it is difficult to imagine anything new being added to it. However, the development of simulation methods has led to the analysis of a few interesting cases that are difficult to analyze analytically. The dynamics of a falling chain is among them.

A detailed and critical review of the history of falling chain problems, in particular of some erroneous approaches to them, has been given recently by Wong and Yasui. ${ }^{1}$ An interesting case is a chain initially gathered in a compact heap located on a table and close to its edge. The motion starts when one of the chain ends is brought over the edge. If we assume that the chain leaves the heap without friction, the model becomes tractable analytically. The surprise is that the acceleration of the chain tip is not, as might be expected, $g$, but $g / 2 .^{2}$ Wong and Yasui ${ }^{1}$ have confirmed the result and review the history of the long lasting erroneous conviction that the acceleration should equal $g / 3$. They locate the source of the error and propose a fool-proof Lagrangian approach to falling chain problems concluding that "Lagrange's method gives definitive answers with unmatched ease, clarity and elegance." They also indicate that the method of Sousa and Rodrigues ${ }^{2}$ is not reliable because it gives an erroneous solution for the falling folded chain.

In the variation of the falling chain problem considered here, the chain is initially attached at both ends to a horizontal support. Then, as one of the ends is released, the chain begins to fall. The case in which the horizontal separation $\Delta x$ between the ends of the chain is zero, that is, the chain is tightly folded, has an analytical solution. According to Wong and Yasui, ${ }^{1}$ the solution was first given by $\mathrm{Hamel}^{3}$ and then repeated by Calkin and March. ${ }^{4}$ The analytical result was confirmed both in experiments ${ }^{4}$ and numerical simulations. ${ }^{5,6}$ We note that Calkin and March also considered a variation of the falling chain problem in which the chain was hanging initially over a smooth horizontal peg and then allowed to slip down one side. ${ }^{7}$ This case in not considered here.

In this paper, we describe the results of experiments analogous to those performed in Ref. 4. In the initial conformation, the ends of the chain of length $L$ are located at the same level but their horizontal separation $\Delta x$ is variable. In the experiments performed in Ref. 4, the time was recorded for the tension acting on the support of the fixed end of the chain to reach its maximum value, that is, the time at which, as they implicitly assumed, the tip of the chain reaches its lowest position. In their study, the largest value of $\Delta x$ was around $0.3 \mathrm{~L}$. In contrast, for our experiments the largest value of $\Delta x$ was $0.999 L$, and we were able to record the entire shape of the consecutive conformations of the falling chain. From the recorded conformations we are able to extract quantitative data for the time dependence of the velocity and acceleration of the chain tip. Calkin and $\mathrm{March}^{4}$ compared their experimental results with the analytical solution of the $\Delta x=0$ model. It is clear that this model is not valid for large $\Delta x$. To overcome this difficulty, we formulated the complete equations of motion for the chain and integrated them numerically, arriving at a quantitative comparison between the experimental and numerical results. We shall demonstrate that the case (which has not been studied before) in which the initial distance between the ends of the chain is very close to $L$, that is, when the chain is initially stretched to its maximum length, is very interesting because the vertical motion of the chain tip becomes identical with the motion of a freely falling body.

\section{ANALYTICAL SOLUTION OF THE FALL OF THE TIGHTLY FOLDED CHAIN}

To understand what we can expect in the experiments with the falling chain, we first consider the case $\Delta x=0$. The complete analysis of this case is in Ref. 4. Thus, we provide only its essential assumptions and results. The basic assumption is that the conformations explored by the falling chain consists of two sections: The falling section of length $L_{a}$, which decreases with time; and the almost motionless section of length $L_{b}$, which increases with time. Such a division of the chain is possible when the initial horizontal separation of the chain ends equals zero and the chain consists of infinitely many short and thin segments. In this limit, the chain can be seen as a tightly folded, perfectly flowing, and infinitely thin continuous filament.

Initially, both ends of the chain are attached to a point of support $O$; the vertical position of which is $y=0$. At time $t=0$, one of the ends of the chain is released and the chain begins to fall. Figure 1 presents the geometry of the system. We assume that the chain has a total length $L$ and its mass $M$ is distributed uniformly along $L$. To simplify the analysis of the results, we introduce the variable $h$ describing the distance of the freely falling tip from its initial position. The $h$ axis is oriented in the direction of the gravitational field. We 


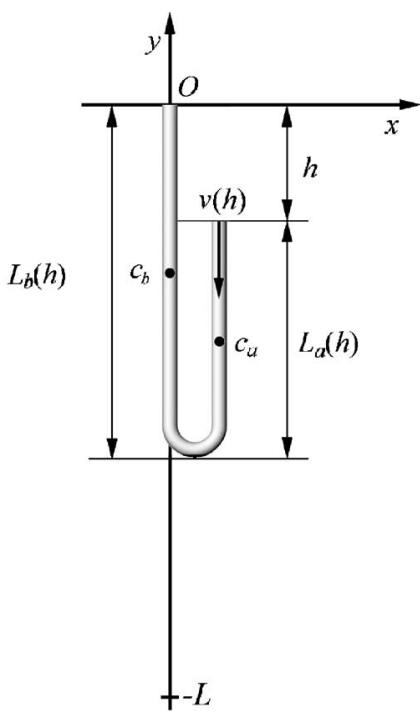

Fig. 1. Geometry of the conformation of the tightly folded chain at time $t>0$. The position of the falling chain is described in terms of $h$. Section (a) of the chain is falling while section (b) is motionless; $c_{a}$ and $c_{b}$ are their centers of mass.

shall refer to $h$ as the fall distance. By assuming that the energy is conserved, it can be shown that the velocity $v_{c}$, acceleration $a_{c}$, and time $t_{c}$ of the chain tip versus the fall distance $h$ are given by:

$$
\begin{aligned}
& v_{c}(h)=\sqrt{\left(1+\frac{L}{L-h}\right) g h,} \\
& a_{c}(h)=\frac{1}{2}\left[1+\left(\frac{L}{L-h}\right)^{2}\right] g, \\
& t_{c}(h)=\int_{0}^{h} \sqrt{\frac{L-s}{g s(2 L-s)}} d s .
\end{aligned}
$$

By analyzing Eqs. (1)-(3), we conclude that both the velocity and the acceleration of the chain tip diverge at a time $t_{c}(L)$ when the tip reaches its lowest position, $h=L$. At small $h$, the velocity and acceleration are approximated by the well-known relations describing the dynamics of free fall:

$$
\begin{aligned}
& v_{b}(h)=\sqrt{2 g h}, \\
& a_{b}(h)=g, \\
& t_{b}(h)=\sqrt{2 h / g} .
\end{aligned}
$$

As calculated in Ref. 4, the time $t_{c}(L)$ at which the tip of the falling folded chain reaches its lowest position equals

$$
t_{c}(L)=0.847213 t_{b}(L),
$$

where $t_{b}(L)$ is the time of the free fall. As shown in Fig. 5 of Ref. 4, the experimentally established time of the chain fall decays with $\Delta x$ in agreement with the theoretical value only at $\Delta x \approx 0.05 L$. In Sec. VI, we shall discuss this result in more detail.

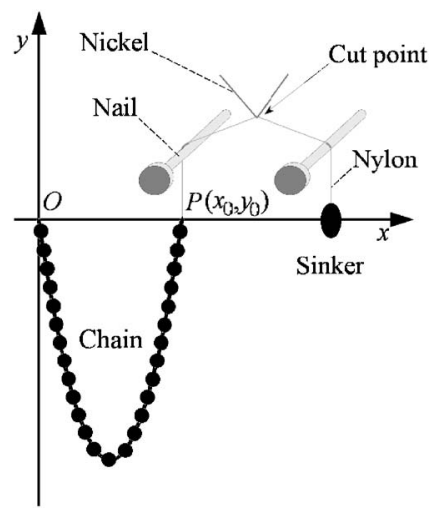

Fig. 2. Schematic of the experimental setup used in this study.

\section{LABORATORY EXPERIMENTS}

The focus of our experimental study is the dynamics of the falling chain, with a particular interest in comparing it to the dynamics of a freely falling weight. To compare the differences in trajectories for the two cases, we designed an experimental setup that makes it possible to record the simultaneous motions of the two objects.

We used a ball chain consisting of stainless-steel identical segments that are made from rods and spheres attached to each other (see Fig. 2). The total length of a segment is $\ell=(4.46 \pm 0.01) \times 10^{-3} \mathrm{~m}$ and the diameter of the spheres is $\phi=(3.26 \pm 0.01) \times 10^{-3} \mathrm{~m}$. In addition, the minimum radius of curvature for which the chain can exist without loading any elastic energy is $R_{\min }=(4.8 \pm 0.2) \times 10^{-3} \mathrm{~m}$. We used a chain of length $L=1.022 \mathrm{~m}$, which corresponds to $n=229$ segments for a total mass of $M=(2.08 \pm 0.01) \times 10^{-2} \mathrm{~kg}$.

The chain is tightly attached at one end to a firm support $O$ by means of a thin thread (see Fig. 2). At the other edge located at point $P=\left(x_{0}, y_{0}\right)$, the chain ends with a rod (we open and remove the last sphere) to which we attach a thin

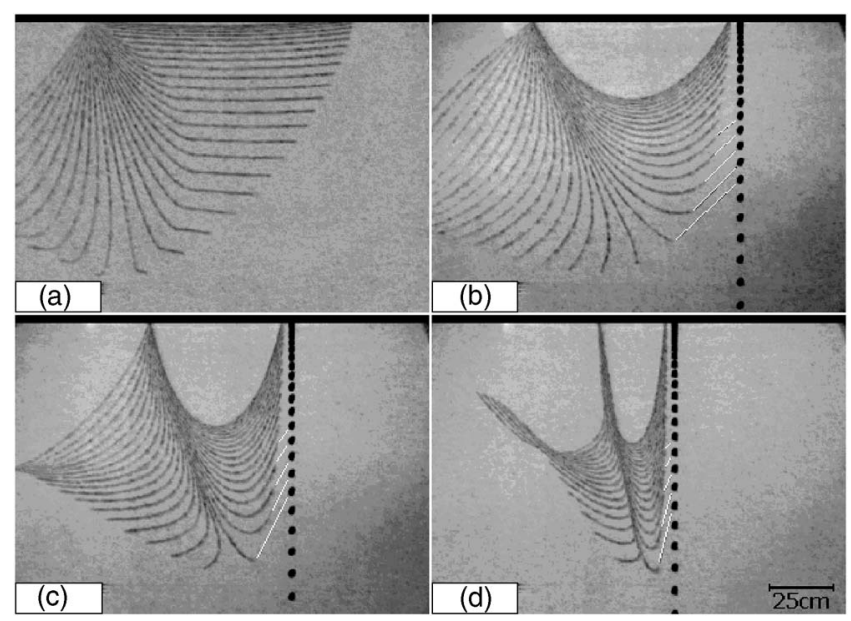

Fig. 3. Successive conformations of the falling chain versus time. The left end of the chain remains attached to the frame, while the right end is free to fall due to gravity. In (b), (c), and (d), white lines have been sketched on the photographic sequence to connect the free falling end of the chain to the freely falling sinker for the last five images before the maximum extension of the chain. The length $L=1.022 \mathrm{~m}$, the time between successive images is $1 / 50 \mathrm{~s}$, and the initial separation between the chain ends is (a) $x_{0}=1.019 \mathrm{~m} \mathrm{(b)} 0.765 \mathrm{~m}$ (c) $0.510 \mathrm{~m}$ and (d) $0.255 \mathrm{~m}$. 


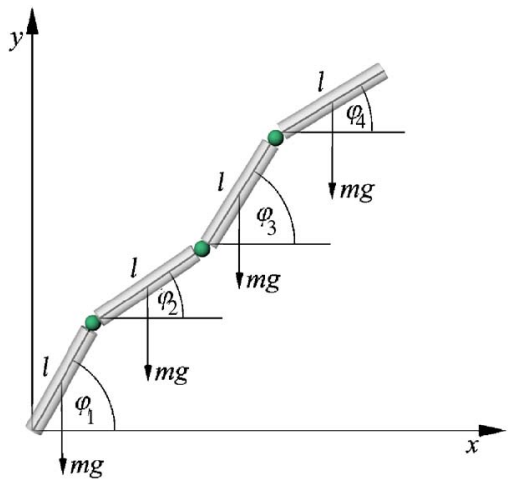

Fig. 4. Schematic representation of the model; $\varphi_{i}$ corresponds to the angle of inclination, $m$ the mass of the chain segment, and $g$ is the gravitational acceleration.

nylon cord (fishing line, diameter $10^{-4} \mathrm{~m}$ ). The free-falling weight (a lead weight used for sinking fishing lines) of mass $\mathcal{M}=10^{-2} \mathrm{~kg}$ is then attached to the other end of the nylon cord (length about $5 \mathrm{~cm}$ ). We then extend the nylon cord between two nails and a thin metallic wire (nickel, diameter $10^{-4} \mathrm{~m}$ ) as sketched in Fig. 2.

The whole system is adjusted to ensure that the sinker and both ends of the chain are at the same level, $y=0$. The setup can be displaced horizontally to vary the initial horizontal separation between the two ends of the chain. Because the mass of the sinker $\mathcal{M}$ is about one-half of the total mass of the chain $M$, the system is almost always equilibrated. (Mechanical equilibrium is further insured by the solid friction in the contact regions of the nylon wire with the nails and the metallic wire.) Thus, the initial conformation formed by the chain after damping of all the disturbances is close to a catenary curve. ${ }^{8}$

Injecting a large electric current (about $1 \mathrm{~A}$ ) through the metallic wire results in cutting suddenly the nylon wire at the point where they make contact. The sinker and the end of the chain then simultaneously start to fall freely under the action of gravity. Note that they both fall with a small piece of nylon cord attached to them. However, as the force that pushes the cord against the nails vanishes, the friction force vanishes as soon as the cord is cut. In addition, during the free fall, the sections of cord have no effect on the dynamics because the mass of nylon is negligible in comparison to the mass of the sinker or chain.

The falling chain and weight are imaged with a standard charge coupled device video camera and the images are recorded on a video cassette recorder. The shutter speed $(1 / 4000 \mathrm{~s})$ is adequate for obtaining clear images of both the chain and sinker (Fig. 3). The filmed sequences of events are digitized by means of a computer equipped with a frame grabber board (Data Translation DT2255). Further analysis with image-processing software (NIH Image) makes it possible to recover 50 images per second from the videos, which are initially made from 25 interlaced images per second. The interlacing allows us to double the time resolution but results in a loss in the spatial resolution, which is typically of about $4 \mathrm{~mm}$ per pixel.

The positions of the falling chain tip and the sinker at consecutive times $t_{i}, i=0,1,2, \ldots$, are determined from the digitized images. To simplify our discussion of the results, the experimentally determined positions of the falling objects are given as the vertical distance $h$ and horizontal distance $w$, which are defined by the deviations of their $x(t)$ and $y(t)$ coordinates from their initial values $\left(x_{0}, y_{0}\right)$ :

$$
\begin{aligned}
& w(t)=x_{0}-x(t), \\
& h(t)=y_{0}-y(t) .
\end{aligned}
$$

We shall refer to $h$ and $w$ as the vertical and horizontal fall distances, respectively. According to their definitions, both falling distances are positive in the initial stages of fall. In all experiments, $y_{0}=0$, and $x_{0}$ was varied in four steps from about $1 \mathrm{~m}$ to $0.25 \mathrm{~m}$. Note that because the motionless end of the chain is attached to the point $(0,0)$, the initial horizontal separation of the chain ends is $\Delta x=x_{0}$, and we denote the initial separation by $x_{0}$. The experimental results are compared to the numerical predictions in Sec. VI.

\section{MODEL OF THE FALLING CHAIN AND ITS EQUATIONS OF MOTION}

We can define several discrete models of the chain; in the following, we present one of them. Its equations of motion will be formulated for the case in which one of the chain ends is attached to the fixed support while the other one is free. Similar models have been considered by other workers. $5,9,10$

The free end of the chain moves under the action of the gravitational field. To simplify the model, we first assume that the chain is constrained to move only in the vertical plane denoted by $(x, y)$. The chain has mass $M$, length $L$, and consists of $n$ thin cylindrical rods (segments) with masses $m_{i}=m=M / n, i=1 \ldots n$, and lengths $\ell_{i}=\ell=L / n$. All segments are considered to be rigid and cannot be deformed. Consecutive segments are connected by joints with friction. Figure 4 shows the geometric representation of our model.

To formulate the equations of motion, generalized coordinates must be specified. Following Ref. 9, we describe the system using angular coordinates indicating the inclination of the consecutive segments with respect to the horizontal $x$ axis.

The position of the first element is determined by the angle $\varphi_{1}$. Similarly, the position of the second element is described by the angle $\varphi_{2} \ldots$. The global conformation of the chain in the plane is uniquely expressed by the angles $\varphi_{i}$, $i=1 \ldots n$.

The Cartesian coordinates of the $i$ th mass center $\left(x_{i}, y_{i}\right)$ can be written as:

$$
\begin{aligned}
x_{i} & =\sum_{j=1}^{i-1} \ell \cos \varphi_{j}+\frac{1}{2} \ell \cos \varphi_{i}, \\
y_{i} & =\sum_{j=1}^{i-1} \ell \sin \varphi_{j}+\frac{1}{2} \ell \sin \varphi_{i} .
\end{aligned}
$$

We use the generalized coordinates $\varphi_{i}$ to derive the Lagrange equations of motion. The motion of the chain is considered as a combination of translational and rotational motions of its segments. Each segment has a moment of inertia $I_{i}=1 / 12 m \ell^{2}$ calculated about the axis perpendicular to the $(x, y)$ plane and passing through the center of mass of the segment. If we take into consideration the relations given in Eq. (9), the kinetic energy of the chain is given by: 


$$
T=\frac{1}{2} \sum_{i=1}^{n}\left[m\left(\dot{x}_{i}^{2}+\dot{y}_{i}^{2}\right)+I_{i} \dot{\varphi}_{i}^{2}\right]
$$

where the dot represents the derivative with respect to the time $t$. The potential energy of the $i$ th segment is given by $m g y_{i}$, where $g$ is the acceleration due to the gravitational field. Thus, the potential energy of the chain may be expressed as

$$
U=\sum_{i=1}^{n} m g y_{i}
$$

To make our model more general, we introduce damping through the Rayleigh dissipation function: ${ }^{11}$

$$
\mathcal{R}=\frac{1}{2} r \sum_{i=1}^{n}\left(\dot{\varphi}_{i}-\dot{\varphi}_{i-1}\right)^{2},
$$

where $r$ is the dissipation coefficient. We assume that the joint that connects the first element of the chain to the support is free of dissipation, which is equivalent to assuming that $\dot{\varphi}_{0}=\dot{\varphi}_{1}$. A similar definition of dissipation has been used. 5,1

The motion of the falling chain is governed by the system of Lagrange equations of the second kind:
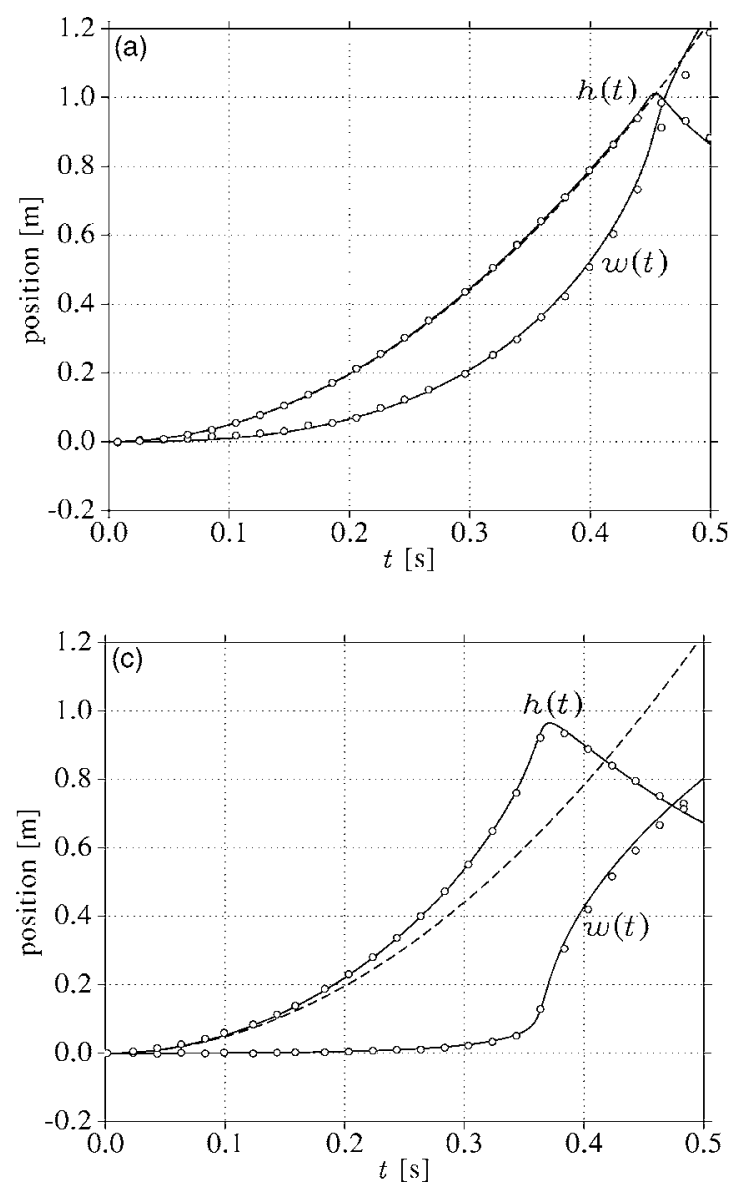

$$
\frac{d}{d t}\left(\frac{\partial \mathcal{L}}{\partial \dot{\varphi}_{i}}\right)-\frac{\partial \mathcal{L}}{\partial \varphi_{i}}+\frac{\partial \mathcal{R}}{\partial \dot{\varphi}_{i}}=0, \quad(i=1 \ldots n),
$$

where $\mathcal{L}=T-U$ is the Lagrangian of the system. By applying Eqs. (10)-(13), we find the set of $n$ equations describing the motion of a chain:

$$
\begin{aligned}
\sum_{j=1}^{n} m_{i, j} c_{i, j} \ddot{\varphi}_{j}= & -\sum_{j=1}^{n} m_{i, j} s_{i, j} \dot{\varphi}_{j}^{2}+\frac{r}{m l^{2}}\left(\dot{\varphi}_{i-1}-2 \dot{\varphi}_{i}+\dot{\varphi}_{i+1}\right) \\
& -\frac{g}{\ell} a_{i} c_{i},
\end{aligned}
$$

where $\quad c_{i}=\cos \left(\varphi_{i}\right), \quad c_{i, j}=\cos \left(\varphi_{i}-\varphi_{j}\right), \quad s_{i, j}=\sin \left(\varphi_{i}-\varphi_{j}\right)$, $a_{i}=n-i+\frac{1}{2}$, and

$$
m_{i, j} \begin{cases}n-i+\frac{1}{3}, & (i=j), \\ n-\max (i, j)+\frac{1}{2}, & (i \neq j) .\end{cases}
$$

\section{NUMERICAL EXPERIMENTS}

The equations of motion in Eq. (14) can be integrated numerically, thus allowing us to simulate the motion of the falling chain. In the presence of dissipation, the resulting system of equations becomes stiff and requires specific numerical methods. We selected the RADAU5 algorithm by
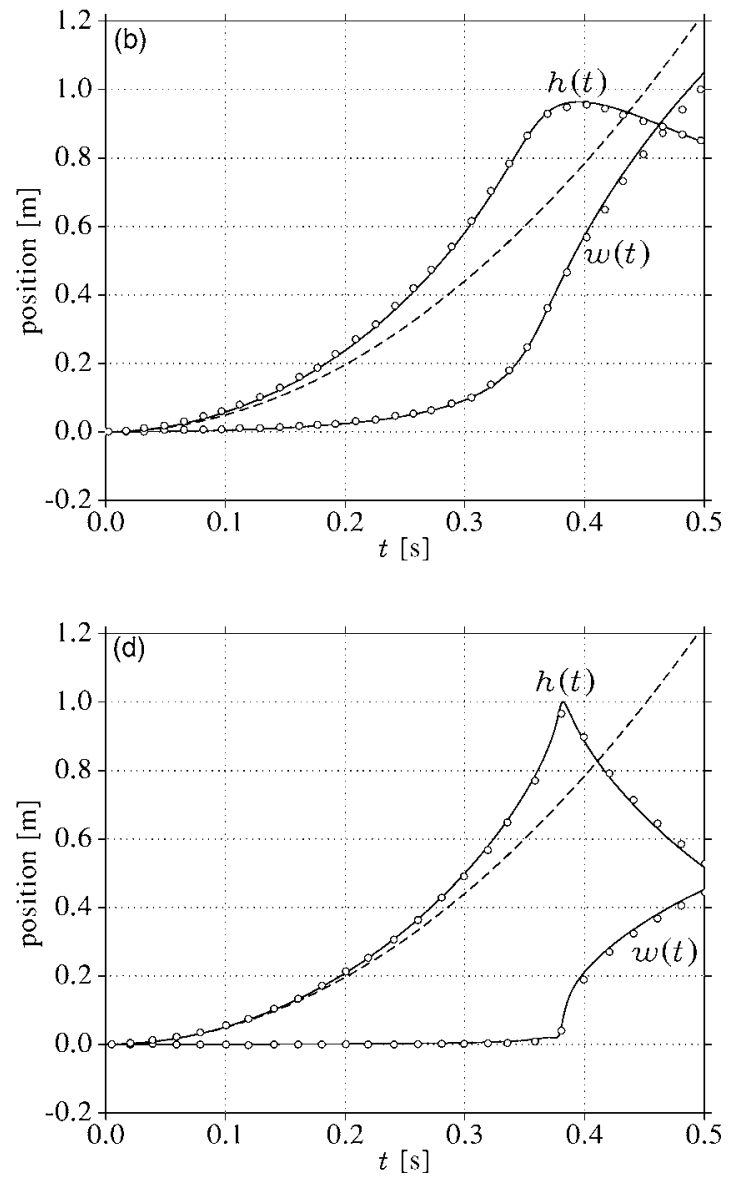

Fig. 5. Comparison of the vertical $h$ and horizontal $w$ fall distances of the falling chain tip found experimentally (circles) and numerically (solid lines). The parabola of the free fall is also shown (dotted lines). The initial separation between the chain ends is the same as in Fig. 3. Numerical data were obtained for $r=2.163 \times 10^{-5} \mathrm{Nm} \mathrm{s}$. The deviations between the numerical and experimental results are given in the middle column of Table I. 
Table I. Deviation between the experimental and numerical results $\delta_{r}$ for three values of the dissipation parameter $r=0,2.163 \times 10^{-5}$, and $r=10^{-4} \mathrm{Nm} \mathrm{s}$.

\begin{tabular}{cccc}
\hline \hline Experiment & $\delta_{0}(\mathrm{~m})$ & $\delta_{2.163 \times 10^{-5}}(\mathrm{~m})$ & $\delta_{10^{-4}}(\mathrm{~m})$ \\
\hline $\mathrm{a}$ & 0.008406 & 0.007672 & 0.01038 \\
$\mathrm{~b}$ & 0.006851 & 0.006654 & 0.00659 \\
$\mathrm{c}$ & 0.006191 & 0.005912 & 0.00669 \\
$\mathrm{~d}$ & 0.008397 & 0.004552 & 0.00991 \\
\hline \hline
\end{tabular}

Hairer and Wanner. ${ }^{12}$ It is based on the implicit Runge-Kutta scheme of order five with the error estimator of order four. ${ }^{13}$

As the initial configuration of the chain, we used the discrete catenary curve shown in Fig. 2 with four separations between the ends of the chain: (a) $x_{0}=1.019 \mathrm{~m}$, (b) $x_{0}=0.765 \mathrm{~m}$, (c) $x_{0}=0.510 \mathrm{~m}$, and (d) $x_{0}=0.255 \mathrm{~m}$, the same values as the separations in the experiments. The simulations were done for a chain with $n=229, L=1.02 \mathrm{~m}$, $M=0.0208 \mathrm{~kg}, g=9.81 \mathrm{~m} / \mathrm{s}^{2}$, and time $t \in[0,0.5] \mathrm{s}$. The only remaining free parameter was the dissipation parameter $r$. We chose a value for $r$ that led to the best agreement of the numerical results with the laboratory experiments.

To compare the numerical results to the experimental data, we monitored the distance between the positions of the chain tip found in consecutive frames of the video recordings and the positions found in the simulations at the same times. The deviation between the laboratory and numerical data obtained in a single experiment is defined as:

$$
\delta=\sqrt{\frac{1}{N} \sum_{i=1}^{N}\left(w_{i}-\hat{w}_{i}\right)^{2}+\left(h_{i}-\hat{h}_{i}\right)^{2}},
$$

where $N$ denotes the number of analyzed frames. The points $\left(w_{i}, h_{i}\right)$ and $\left(\hat{w}_{i}, \hat{h}_{i}\right)$ for $i=1 \ldots N$ are the horizontal and vertical deviation from the initial position of the chain tip found in consecutive frames of the laboratory and numerical experiments, respectively. To find the optimal value of $r$ for all four experiments, we determined the total distance

$$
\Delta=\delta^{(a)}+\delta^{(b)}+\delta^{(c)}+\delta^{(d)} .
$$

We investigated the values of $\Delta$ for $R$ from $r_{1}=0$ to $r_{2}=10^{-4} \mathrm{Nm} \mathrm{s}$. The optimal value of $r$ found using a leastsquares algorithm based on the SVDFIT procedure ${ }^{14}$ was found to be $r=2.163 \times 10^{-5} \mathrm{Nm} \mathrm{s} ; \Delta$ reaches its minimum value $\Delta(r)=0.02479 \mathrm{~m}$. At the ends of the analyzed range of $r$, we found $\Delta\left(r_{1}\right)=0.029845 \mathrm{~m}$ and $\Delta\left(r_{2}\right)=0.03357 \mathrm{~m}$. Table I summarizes how well the data for each of the four experimental cases is fit by the optimal value of $r$.

Figure 5 provides a further comparison between the simulations and the experimental results. We see excellent agreement between the simulated and experimental data.
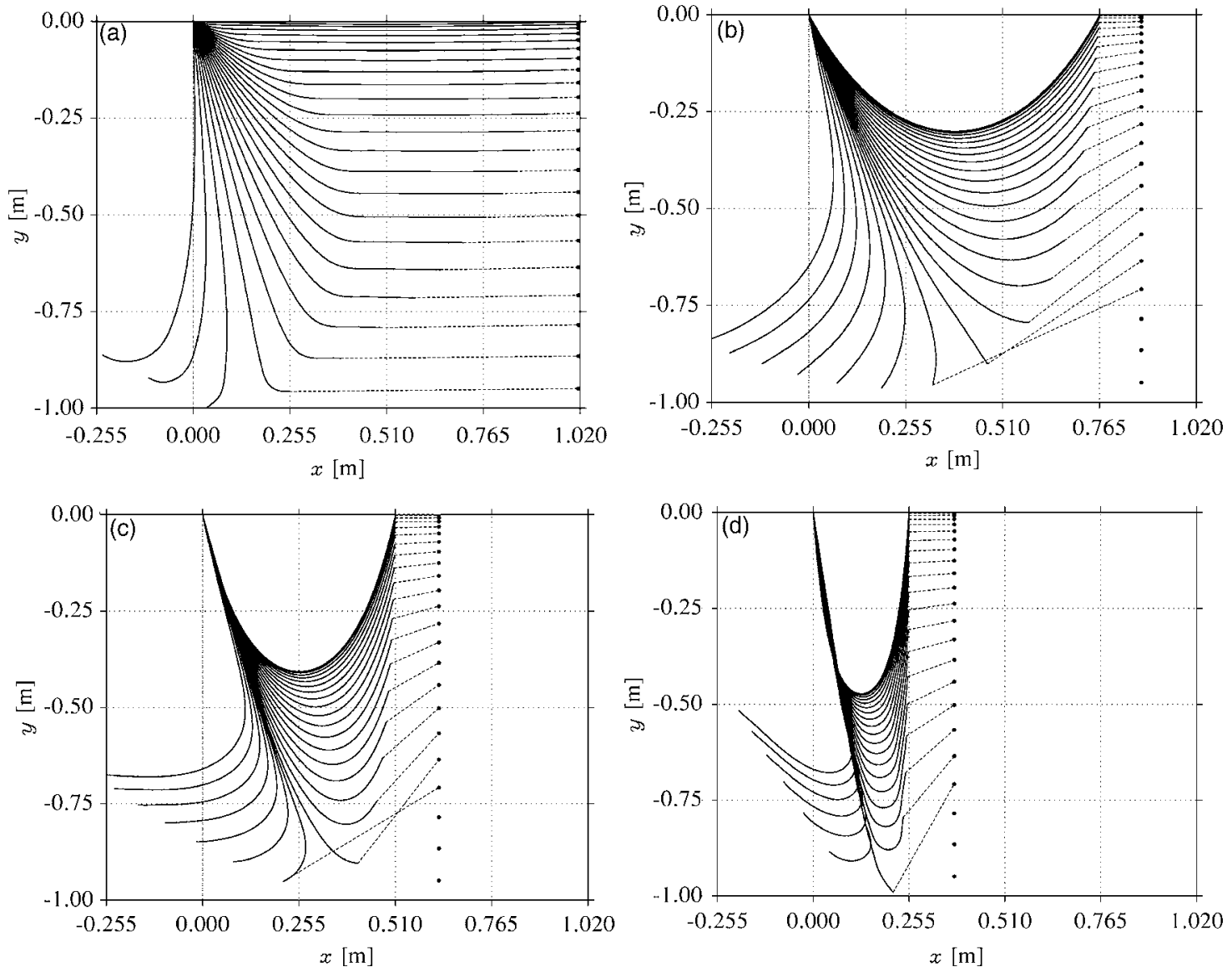

Fig. 6. Successive conformations of the falling chain versus time found in the simulations. The latter were performed with $n=229$, $L=1.02 \mathrm{~m}$, $M=0.0208 \mathrm{~kg}$, and $r=2.163 \times 10^{-5} \mathrm{Nm} \mathrm{s}$, for which the numerical solutions of the equations of motion show the best fit to the experimental results. The initial conformations of the chain were discrete catenary curves with the same as in Fig. 3. The positions of the freely falling body are shown on the right-hand sides of the figures; dotted lines connect them with the respective positions of the tip of the falling chain. 

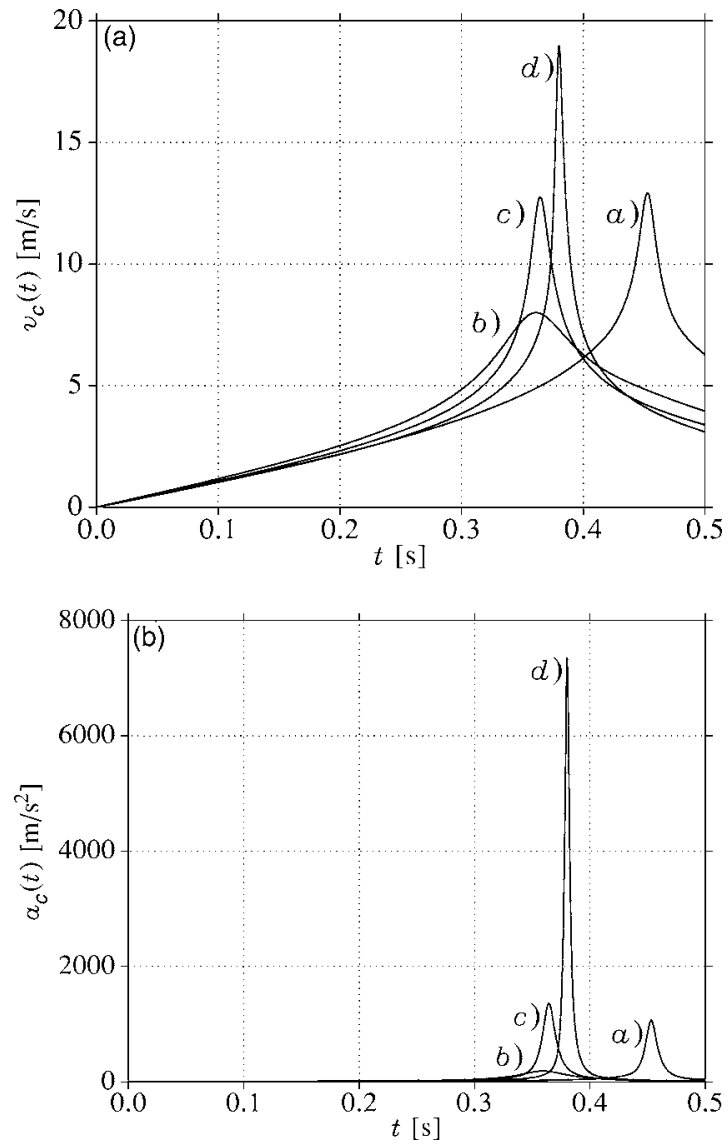

Fig. 7. Evolution of the numerically determined moduli of the velocity and the acceleration of the falling chain tip. The initial separation of the chain ends are given in Fig. 3 and $r=2.163 \times 10^{-5} \mathrm{Nm} \mathrm{s}$.

The consecutive conformations of the falling chain found in the simulations are presented in Fig. 6. These conformations correspond to the same times as the laboratory experiments. The positions of the freely falling body are also shown. By comparing Figs. 3 and 6, we see that the shapes of the experimental and numerical conformations are nearly identical.

\section{QUANTITATIVE ANALYSIS}

Because of the excellent agreement between the experimental data and the simulations, we use the data from the numerical simulations to analyze the details of the falling chain dynamics.

We first analyze the relation between the time dependence of the vertical fall distances of the chain tip and the falling sinker (Fig. 5). Note that in case (a), where the initial conformation of the chain is straight and horizontal, the vertical fall of the chain tip and the falling sinker are identical up to the time at which, having reached its maximum vertical fall distance, the tip starts moving up. This observation becomes clear when we notice that during the fall the chain end remains horizontal-its vertical motion must thus be identical with the falling sinker. The chain end remains horizontal because the chain displays no elasticity and no energy is stored in the bent regions. This phenomenon, found both in the laboratory experiments and confirmed by the simulations,
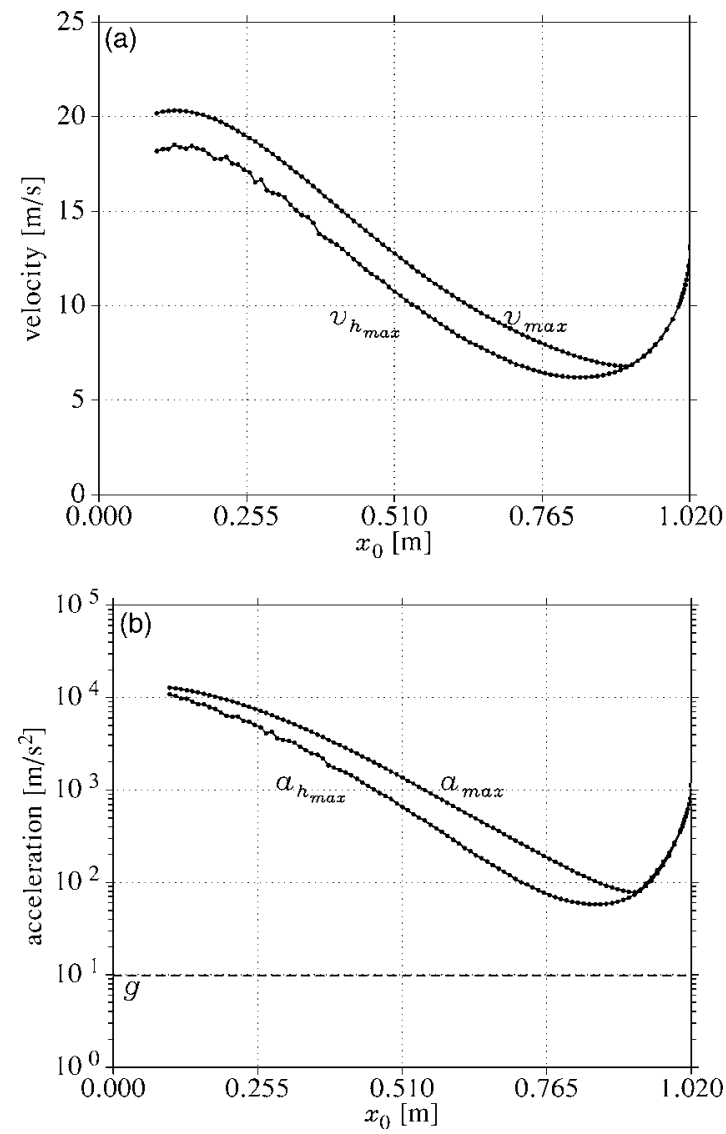

Fig. 8. Moduli of the (a) velocity and (b) the acceleration of the chain tip versus the initial horizontal separation of the chain ends. $v_{\max }$ and $a_{\max }$ are, respectively, the maximum velocity and acceleration reached by the chain tip during its fall. $v_{h_{\max }}$ and $a_{h_{\max }}$ are the velocity and acceleration of the chain tip observed at the time at which the tip reaches its lowest position. Figure $8($ b) is plotted with a logarithmic scale. The gravitational acceleration $g$ is marked with a dashed line. The plots were obtained numerically for $r=2.163 \times 10^{-5} \mathrm{Nm} \mathrm{s}$.

suggests the existence of an approximate analytical treatment of the problem. However, we have thus far not been able to formulate an analytical solution.

In cases (b), (c), and (d) the vertical fall distance of the chain tip, up to the time $t_{h_{\max }}$ at which the vertical fall distance of the chain tip reaches its maximum value $h_{\max }$, is seen to always be ahead of the vertical fall distance of the sinker. This observation is sometimes summarized by the general statement that the chain falls faster than a body.

To further understand this behavior, we analyzed the time dependence of the velocity $v_{c}$ and the acceleration $a_{c}$ of the chain tip. To do so, we performed a series of simulations with $x_{0}$ in the range $[0.1, \ldots, 1.02] \mathrm{m}$. All other parameters of the simulation were the same as defined in Sec. V. The smallest value of the initial separation $x_{0}$ was equal to $0.1 \mathrm{~m}$ because smaller initial separations produced very complex chain-fall dynamics. This behavior was seen in both the simulations and laboratory experiments. Note that by velocity and acceleration we mean here the moduli of the velocity and acceleration vectors.

Figure 7 plots the velocity and the acceleration versus time for four initial spacings $x_{0}$. Two characteristic features in these plots are the peak heights and the times at which these maxima occur. In Fig. 8(a), the dependence of $v_{\max }$ on $x_{0}$ and 

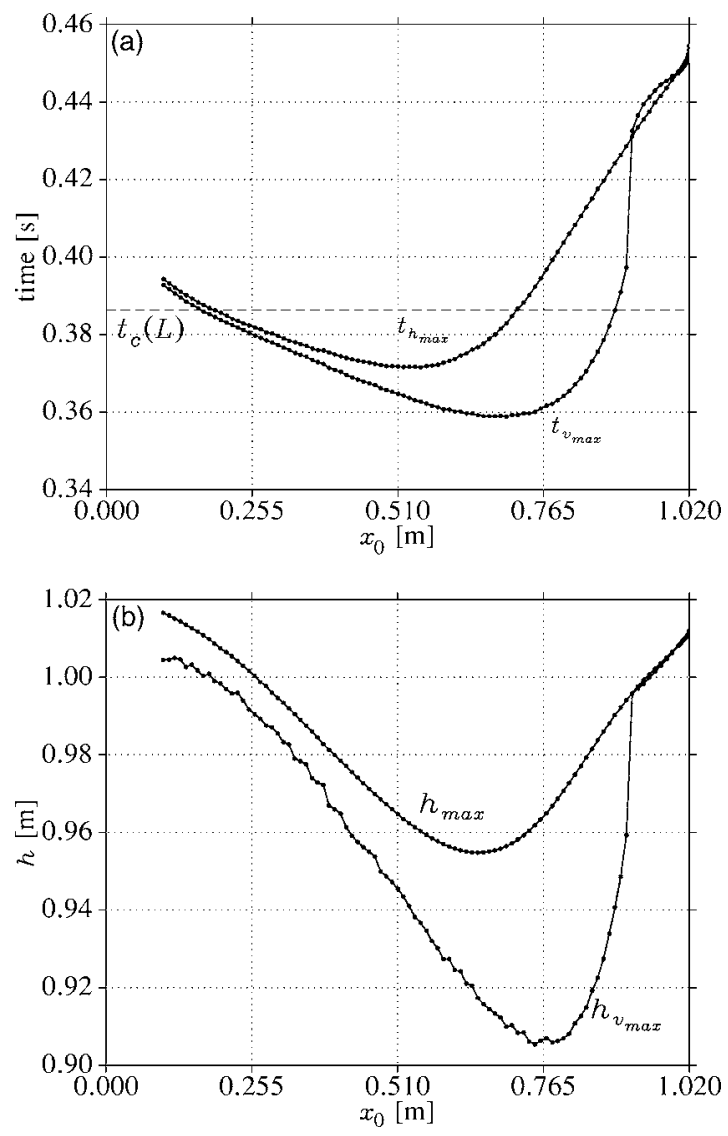

Fig. 9. (a) Characteristic times, $t_{h_{\max }}$ and $t_{v_{\max }}$ versus the initial separation $x_{0}$. $t_{h_{\max }}$ is the time at which the chain tip reaches its lowest position; $t_{v_{\max }}$ is the time at which it reaches its maximum velocity $v_{\max }$. The dashed line represents the time $t_{c}(L) \approx 0.386722$ at which the velocity diverges in the analytical model considered in Sec. II. (b) Characteristic distances $h_{\max }$ and $h_{v_{\max }}$ versus the initial separation $x_{0} . h_{\max }$ is the largest vertical fall distance reached by the chain tip; $h_{v_{\max }}$ is the vertical fall distance of the chain tip at which it reaches its maximum velocity. The plots were obtained numerically with $r=2.163 \times 10^{-5} \mathrm{Nm} \mathrm{s}$.

$v_{h_{\max }}$ (that is, the velocity of the chain tip at its lowest position $h_{\text {max }}$ ) on $x_{0}$ is displayed. At small $x_{0}$, there exists a short interval within which $v_{\max }$ slightly increases reaching its global maximum at $x_{0} \approx 0.1314 \mathrm{~m}$. Then, over a broad interval, $v_{\max }$ decreases and reaches its global minimum value at $x_{0} \approx 0.9040 \mathrm{~m}$. Up to this value of $x_{0}$, the velocity $v_{h_{\max }}$ is smaller than the maximum velocity $v_{\max }$. For $x_{0}>0.9040 \mathrm{~m}$, when the maximum extension of the chain is approached, the two velocities become practically equal.

As Fig. 9(a) shows, the time $t_{v_{\max }}$ at which the velocity of the chain tip reaches its maximum value precedes in general the time $t_{h_{\max }}$ at which the chain tip reaches its lowest position $h_{\max }$. The lowest position of the chain tip is reached fastest for $x_{0} \approx 0.5500 \mathrm{~m}$, that is, when the initial horizontal distance between the chain ends is approximately one-half of its total length, and $t_{h_{\max }}$ is a maximum when the chain is initially straight. Figure 9(a) also compares the time $t_{v_{\max }}$ of the maximum velocity and the time $t_{c}(L)$ of the velocity divergence found in the solvable model discussed in Sec. II. As seen in Fig. 9(a), the two times are close to each other at the values of $x_{0}$ at which $v_{\max }$ reaches its global maximum and minimum. In the range of $x_{0}$ located between the two values, $t_{v_{\max }}$ is less than $t_{c}(L)$.
Figure 9(b) gives the relation between the maximum fall distance of the chain tip $h_{\max }$, and the fall distance $h_{v_{\max }}$ at which the tip reaches its maximum velocity as a function of the initial separation of the chain ends. We can clearly see that, in general, the maximum velocity is reached before the chain tip reaches its maximum fall distance. We note that for all the values of $x_{0}$ studied, the maximum fall distance never reaches the theoretically possible value $L$, although as the plot of $h_{\max }$ versus $x_{0}$ suggests, it tends to $L$ as $x_{0} \rightarrow 0$.

The last question we have addressed concerning the velocity data is the correlation between the value of the peak velocity $v_{\max }$ and the time $t_{v_{\max }}$ at which it is reached. As we have demonstrated, the peak of the velocity is a maximum at a small initial separation of the chain ends, but we should not conclude that the peak is reached in the shortest time. As seen in Fig. 9(a), the initial separation of the chain ends at which the velocity peak is reached most quickly occurs at $x_{0} \approx 0.7000 \mathrm{~m}$.

Plots of the acceleration of the chain tip versus the time for a few values of the initial separation $x_{0}$ are given in Fig. 7(b). As for the velocity plots, we observe distinct peaks. Figure 8(b) demonstrates that the highest peak in the acceleration is observed at the smallest initial separation of the chain ends. Its value for the experimentally studied case of $x_{0}=0.255 \mathrm{~m}$ is $7352 \mathrm{~m} / \mathrm{s}^{2}$, which is about 40 times larger than the value observed at $x_{0}=0.765 \mathrm{~m}$, where it equals $186.3 \mathrm{~m} / \mathrm{s}^{2}$. That such large values of the acceleration are realistic was demonstrated by Krehl et al. ${ }^{15}$ who studied the dynamics of a cracking whip. What is the relation between the dynamics of the falling chain and the whip? ${ }^{16,17}$ The two systems seem to be completely different because, in the cracking whip problem, the gravitational forces are usually neglected. However, the end of the folded whip attached to the whip handle is subject to a strong acceleration. If we change the laboratory reference frame to the noninertial frame moving with the end of the handle, then strong inertial forces equivalent to the gravitational forces are introduced into the system. Thus, our conclusions about the falling chain are also applicable to an accelerated whip. In particular, there exists a special conformation to which the whip should be brought before its handle starts accelerating, such that the whip tip will eventually reach the maximum velocity, exceeding the speed of sound velocity, thus allowing one to produce the crack sound.

Results of the laboratory experiments and simulations also show that, in contrast to what is observed in the simplified model of the tightly folded chain, the acceleration $a_{h_{\max }}$ that the chain tip reaches at its lowest position $h_{\max }$ is not the maximum acceleration $a_{\max }$ as shown in Fig. 8(b).

\section{SUMMARY AND DISCUSSION}

The experimental and numerical work reveals new and interesting facts concerning the dynamics of the falling chain.

1. The time dependence of the velocity and acceleration displays distinct peaks whose heights depend on the initial separation of the chain ends. The highest peaks are observed for small initial separations. There is an approximate analytical theory of tightly folded chain dynamics that explains the origin of the rapid increase of the velocity and acceleration. However, the theory predicts that 
both the velocity and the acceleration diverge and is unable to predict the finite height of the peaks.

2. For our system, the velocity peak is highest at $x_{0}=0.1288 L$, and its amplitude is smallest for $x_{0}=0.8863 \mathrm{~L}$.

3. For the case in which the initial separation of the chain ends is largest, the dynamics of the vertical fall of the chain tip is identical with the dynamics of free fall. This coincidence corresponds with the fact that the end section of the chain remains horizontal during the fall. This observation suggests the existence of an approximate analytical treatment.

4. The time at which the chain tip reaches its maximum velocity generally comes before the time at which it reaches its lowest vertical position except if the initial separation of the chain ends is larger than $0.8863 \mathrm{~L}$.

5. The ratio of the amplitudes of the highest and smallest acceleration peaks is about 166.5 , which is unexpectedly large. The appearance of the high acceleration peaks may have some practical implications, because at the time when the acceleration reaches its highest value, the force acting on the chain tip also becomes very large and may lead to damage of the chain.

Our experiments indicate that when the initial conformation of the chain is tightly folded, the vertical acceleration of the falling chain tip is always higher than $g$. The explanation is given by energy conservation. The falling chain is divided into two parts: The almost motionless part attached to the support and the moving part. The falling chain lowers its potential energy on the account of the kinetic energy of the continuously shorter, moving part of the chain. Because the mass of the latter decreases, its velocity grows faster than does the velocity of a compact falling body whose mass is constant in time. A falling rope exhibits even more interesting behavior, because dissipation plays a much more important role and elasticity becomes a crucial factor.

\section{ACKNOWLEDGMENTS}

The authors thank S. Breiter for helpful discussions and V. Bergeron for his critical reading of the manuscript.

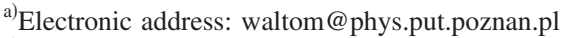

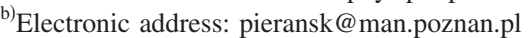

${ }^{c)}$ Electronic address: jean-christophe.geminard@ens-lyon.fr

${ }^{1}$ C. W. Wong and K. Yasui, "Falling chains," arXiv.org:physics 0508005.

${ }^{2}$ C. A. de Sousa and V. H. Rodrigues, "Mass redistribution in variable mass systems," Eur. J. Phys. 25, 41-49 (2004).

${ }^{3} \mathrm{G}$. Hamel, Theoretische Mechanik, 2nd ed. (Springer-Verlag, Berlin, 1949).

${ }^{4}$ M. G. Calkin and R. H. March, "The dynamics of a falling chain: I," Am. J. Phys. 57, 154-157 (1989).

${ }^{5}$ M. Schagerl, A. Steindl, W. Steiner, and H. Troger, "On the paradox of the free falling folded chain," Acta Mech. 125, 155-168 (1997).

${ }^{6} \mathrm{~W}$. Tomaszewski and P. Pieranski, "Dynamics of ropes and chains. I. The fall of the folded chain," New J. Phys. 7, 45-61 (2005).

${ }^{7}$ M. G. Calkin, "The dynamics of a falling chain: II," Am. J. Phys. 57, 157-159 (1989)

${ }^{8}$ Strictly speaking, the initial conformation formed in the experiments with chains built from a finite number of segments is not a catenary curve. We can understand that this is the case by considering the conformation of a chain built from an odd number of segments whose ends are fixed to points located at the same level and separated in the horizontal direction by the length of a single segment. In this case, the initial conformation consists of two exactly vertical pieces. This conformation was used by Schagerl in his laboratory experiments.

${ }^{9}$ J. M. Robson, "The physics of fly casting," Am. J. Phys. 58, 234-240 (1990).

${ }^{10}$ J. Galán, W. B. Fraser, D. J. Acheson, and A. R. Champneys, "The parametrically excited upside-down rod: An elastic jointed pendulum model," J. Sound Vib. 280, 359-377 (2005).

${ }^{11}$ H. Goldstein, Classical Mechanics, 2nd ed. (Addison-Wesley, Reading, MA, 1980).

${ }^{12}$ See $\langle$ http://www.unige.ch/ hairer/software.html $\rangle$.

${ }^{13}$ E. Hairer and G. Wanner, Solving Ordinary Differential Equations II: Stiff and Differential Algebraic Problems, 2nd ed. (Springer-Verlag, Berlin, 1996).

${ }^{14}$ W. H. Press, B. P. Flannery, S. A. Teukolsky, and W. T. Vetterling, $N u$ merical Recipes in $C$, The Art of Scientific Computing, 2nd ed. (Cambridge University Press, Cambridge, U.K., 1992).

${ }^{15}$ P. Krehl, S. Engemann, and D. Schwenkel, "The puzzle of whip crackinguncovered by a correlation of whip-tip dynamics with shock wave emission," Shock Waves 8, 1-9 (1998).

${ }^{16}$ A. Goriely and T. McMillen, "Shape of a cracking whip," Phys. Rev. Lett. 88(24), 244301-1-4 (2002).

${ }^{17}$ T. McMillen and A. Goriely, "Whip waves," Physica D 184, 192-225 (2003).

\section{COSMIC REALITY VS. MICROSCOPIC REALITY}

Imagine yourself out some evening with a particularly close friend. You stare upward at the vast open spaces of the evening sky and gaze at the myriad twinkling stars that spread themselves across the void. "Ah," you say, "just look at those stars; isn't space enthralling!" It may very well be, but space isn't what you are looking at. You are, in fact, looking at time! None of the stars that have you so enraptured are even there, where you now see them. Every twinkling dot is light that was sent out by that star millions and billions of years ago. What is up there tonight, or should I say what your brain projects upward as being "up there," is a vast illusion, a collage of space and time, made up of light from objects that are nowhere near where they appear to be. Illusions such as this require vast distances and times in order to come into being. They belong to cosmic reality. Such illusions do not exist in microscopic reality, but others equally interesting do. It is just one example of how we can be fooled when we intrude on another reality with a mind made for this reality.

Morton Tavel, Contemporary Physics and the Limits of Knowledge (Rutgers, 2002), pp. 25-26. 